\title{
ПРЕДЕЛЬНЫЕ ТЕОРЕМЫ ДЛЯ ПОСЛЕДОВАТЕЛЬНОСТИ СЕРИЙ ОДНОМЕРНЫХ СЛУЧАЙЫХ ВЕЛИЧИН С ПРОИЗВОЛЬНЫМ ХАРАКТЕРОМ ЗАВИСИМОСТИ
}

Пусть имеется последовательность серий случайных величин

$$
\xi_{n 0}, \xi_{n 1}, \xi_{n 2}, \ldots, \xi_{n k_{n}},
$$

причем $0=t_{n 0}<t_{n 1}<\ldots<t_{n k_{n}}=1-$ разбиение отрезка $[0,1]$ такое, что $\lambda_{n}=\max _{k}\left[t_{n k+1}-t_{n k}\right] \rightarrow 0, n \rightarrow \infty$.

Введем обозначения:

$$
\begin{aligned}
& \Delta t_{n k}=t_{n k+1}-t_{n k} ; \\
& \Delta \xi_{n k}=\xi_{n k+1}-\xi_{n k} ;
\end{aligned}
$$

$F_{n k}$ - минимальная $\sigma$-алгебра, образованная случайными величинами $\left[{ }^{1}\right] ; \alpha_{n k}, \beta_{n k}-$ некоторые случайные величины. И пусть

$$
\begin{gathered}
M\left(\Delta \xi_{n k} / F_{n k}\right)=\alpha_{n k} \Delta t_{n k}, \\
M\left(\left[\Delta \xi_{n k}\right]^{2} / F_{n k}\right)=\beta_{n k}^{2} \Delta t_{n k}+\left(\alpha_{n k} \Delta t_{n k}\right)^{2} .
\end{gathered}
$$

Т ео ре м а 1. Если существуют неслучайные функции $a_{n}(x), \sigma_{n}(x)$, для которых при любом $N>0$

$$
\begin{gathered}
\limsup _{n \rightarrow \infty} M\left[\frac{\alpha_{n k}-a_{n}\left(\xi_{n k}\right)}{\sigma_{n}\left(\xi_{n k}\right)}\right]^{4} \chi_{N}\left(\xi_{n k}\right)=0, \\
\lim _{n \rightarrow \infty}\left[1+\sup _{\left|f_{n}(x)\right| \leqslant N}\left(\frac{a_{n}(x)}{\sigma_{n}(x)}\right)^{4}\right] \sup _{k} M\left[\frac{\beta_{n k}-\sigma_{n}\left(\xi_{n k}\right)}{\sigma_{n}\left(\xi_{n k}\right)}\right]^{4} \chi_{N}\left(\xi_{n k}\right)=0,
\end{gathered}
$$

где

$f_{n}(x)=\int_{0}^{x} \exp \left\{-2 \int_{0}^{u} \frac{a_{n}(v)}{\sigma_{n}^{2}(v)} d v\right\} d u, \xi_{n k}=f_{n}\left(\xi_{n k}\right), \chi_{N}(x)-$ индикатор отрезка $[-N, N]$, и если имеют место условия 1) для произвольной постоянной $N>0$

$$
\begin{gathered}
f_{n}^{\prime}(x) \sigma_{n}(x) \leqslant K_{N}, \quad \sigma_{n}(x)>0, \\
\lim _{n \rightarrow \infty}\left\{\lambda_{n} \sup _{\left|f_{n}(x)\right| \leqslant N}\left|\frac{a_{n}(x)}{\sigma_{n}(x)}\right|^{3}+\left(\sup _{\left|f_{n}(x)\right| \leqslant N}\left|\frac{a_{n}(x)}{\sigma_{n}(x)}\right|^{2}+1\right) \times\right. \\
\left.\times \sum_{i=0}^{k_{n}-1} M\left[\frac{\Delta \xi_{n i}}{\sigma_{n}\left(\xi_{n i}\right)}\right]^{h} \chi_{N}\left(\xi_{n i}\right)\right\}=0,
\end{gathered}
$$




$$
\lim \sum_{i=0}^{k_{n}-1} M\left|\int_{\xi_{n i}}^{\xi_{n i+1}} \int_{\xi_{n i}}^{x}\left[f_{n}^{\prime \prime}(u)-f_{n}^{\prime \prime}\left(\xi_{n i}\right)\right] d u d x\right| \chi_{N}\left(\xi_{n i}\right)=0
$$

кроме того, существует функция $\sigma_{0}(x)$, непрерывная всюду, за исключением, может быть, только конечного числа разрывов 1-го рода в каждой ограниченной области $0<\delta \leqslant \sigma_{0}^{2}(x) \leqslant K\left[1+|x|^{2}\right] u$

$$
\lim _{n \rightarrow \infty} \sum_{i=0}^{k_{n}-1} M\left|f_{n}^{\prime}\left(\xi_{n i}\right) \sigma_{n}\left(\xi_{n i}\right)-\sigma_{0}\left(\zeta_{n i}\right)\right|^{2} \chi_{N}\left(\zeta_{n i}\right) \Delta t_{n i}=0,
$$

2) $\sup _{n} P\left\{\sup _{i}\left|\zeta_{n i}\right|>N\right\} \rightarrow 0, N \rightarrow \infty$,

а распределение случайной величины $\xi_{n 0}$ сходится при $n \rightarrow \infty \kappa$ распре-

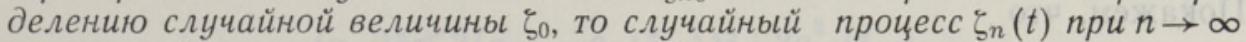
слабо сходится к решению уравнения Нто

$$
\zeta(t)=\zeta_{0}+-\int_{0}^{t} \sigma_{0}(\zeta(s)) d w(s)
$$

и для всякого непрерывного на $C_{[0,1]}$ функционала $F$ распределение $F\left(\zeta_{n}(t)\right)$ сходится $\kappa$ распределению $F(\zeta(t))$.

Док а з а тельст во. Введем величину

$$
\omega_{n k}=\frac{\Delta \xi_{n k}-\alpha_{n k} \Delta t_{n k}}{\beta_{n k}} .
$$

Тогда $\Delta \xi_{n k}$ представима в виде

$$
\Delta \xi_{n k}=\alpha_{n k} \Delta t_{n k}+\beta_{n k} \omega_{n k},
$$

и можно записать

$$
\begin{gathered}
\xi_{n k+1}=f_{n}\left(\xi_{n k+1}\right)=\xi_{n 0}+\sum_{i=0}^{k} f_{n}^{\prime}\left(\xi_{n i}\right) \Delta \xi_{n i}+ \\
+\frac{1}{2} \sum_{i=0}^{k} f_{n}^{\prime \prime}\left(\xi_{n i}\right)\left[\Delta \xi_{n i}\right]^{2}+\sum_{i=0}^{k} \int_{\xi_{n i}}^{\xi_{n i+1}} \int_{n i}^{x}\left[f_{n}^{\prime \prime}(u)-f_{n}^{\prime \prime}\left(\xi_{n i}\right)\right] d u d x .
\end{gathered}
$$

Далее, обозначим

$$
R_{n}=\sum_{i=0}^{k} \int_{\xi_{n i}}^{\xi_{n i+1}} \int_{\xi_{n i}}^{x}\left[f_{n}^{\prime \prime}(u)-f_{n}^{\prime \prime}\left(\xi_{n i}\right)\right] d u d x
$$

Учитывая (12) и тот факт, что в силу вида функции $f_{n}(x)$ имеет место соотношение

$$
f_{n}^{\prime}(x) a_{n}(x)+1 / 2 f_{n}^{\prime \prime}(x) \sigma_{n}^{\prime \prime}(x)=0
$$

получаем:

$$
\xi_{n k+1}=\zeta_{n 0}+\sum_{i=0}^{k} f_{n}^{\prime}\left(\xi_{n i}\right)\left[\alpha_{n i}-a_{n}\left(\xi_{n i}\right)\right] \Delta t_{n i}+\sum_{i=0}^{k} f_{n}^{\prime}\left(\xi_{n i}\right) \times
$$




$$
\begin{aligned}
\times \beta_{n i} \omega_{n i}+ & \frac{1}{2} \sum_{i=0}^{k} f_{n}^{\prime \prime}\left(\xi_{n i}\right)\left(\alpha_{n i} \Delta t_{n i}\right)^{2}+\sum_{i=0}^{k} f_{n}^{\prime \prime}\left(\xi_{n i}\right) \alpha_{n i} \beta_{n i} \omega_{n i} \Delta t_{n i}+ \\
& +\frac{1}{2} \sum_{i=0}^{k} f_{n}^{\prime \prime}\left(\xi_{n i}\right)\left[\beta_{n i}^{2}-\sigma_{n}^{2}\left(\xi_{n i}\right)\right] \Delta t_{n i}+ \\
& +\frac{1}{2} \sum_{i=0}^{k} f_{n}^{\prime \prime}\left(\xi_{n i}\right) \beta_{n i}^{2}\left(\omega_{n i}^{2}-\Delta t_{n i}\right)+R_{n}
\end{aligned}
$$

Оценим величину

$$
I_{1}=\sum_{i=0}^{k} f_{n}^{\prime}\left(\xi_{n i}\right)\left[\alpha_{n i}-a_{n}\left(\xi_{n i}\right)\right] \Delta t_{n i} .
$$

Покажем, что

$$
P\left\{\sup _{k}\left|I_{1}\right|>\varepsilon\right\} \rightarrow 0, \quad n \rightarrow \infty .
$$

Действительно,

$$
\begin{aligned}
& P\left\{\sup _{k}\left|I_{1}\right|>\varepsilon\right\}=P\left\{\sup _{k} \mid \sum_{i=0}^{k} \chi_{\left|\xi_{n i}\right| \leqslant N} f_{n}^{\prime}\left(\xi_{n i}\right)\left[\alpha_{n i}-a_{n}\left(\xi_{n i}\right)\right] \Delta t_{n i}+\right. \\
& \left.+\sum_{i=0}^{k} \chi_{\left|\xi_{n i}\right|>N} f_{n}^{\prime}\left(\xi_{n i}\right)\left[\alpha_{n i}-a_{n}\left(\xi_{n i}\right)\right] \Delta t_{n i} \mid>\varepsilon\right\} \leqslant \\
& \leqslant P\left\{\sup _{k}\left|\zeta_{n k}\right|>N\right\}+\frac{2}{\varepsilon} M\left(\sum_{i=0}^{k_{n}-1} \chi_{\left|\xi_{n i}\right| \leqslant N} \mid f^{\prime}{ }_{n}\left(\xi_{n i}\right) \times\right. \\
& \left.\times\left[\alpha_{n i}-a_{n}\left(\xi_{n i}\right)\right] \mid \Delta i_{n i}\right) \leqslant P\left\{\sup _{k}\left|\zeta_{n k}\right|>N\right\}+ \\
& +\frac{2}{\varepsilon} K_{N} \sup _{0 \leqslant i \leqslant k_{n}}\left(M\left[\frac{\alpha_{n i}-a_{n}\left(\xi_{n i}\right)}{\sigma_{n}\left(\xi_{n i}\right)}\right]^{4} \chi_{\left|\xi_{n}\right| \leqslant N}\right)^{1 / 4} \times \\
& X_{i=0}^{k_{n}-1} \Delta t_{n i} \rightarrow 0, \quad n \rightarrow \infty, \quad t \in[0,1] .
\end{aligned}
$$

Итак, (16) доказано. Рассмотрим теперь величину

$$
\begin{gathered}
I_{n k}=\sum_{i=0}^{k} f^{\prime}{ }_{n}\left(\xi_{n i}\right)\left(\beta_{n i}+\sigma_{n}\left(\xi_{n i}\right)-\sigma_{n}\left(\xi_{n i}\right)\right) \omega_{n i}= \\
=\sum_{i=0}^{k} f_{n}^{\prime}\left(\xi_{n i}\right) \sigma_{n}\left(\xi_{n i}\right) \omega_{n i}+\sum_{i=0}^{k} f^{\prime}{ }_{n}\left(\xi_{n i}\right)\left[\beta_{n i}-\sigma_{n}\left(\xi_{n i}\right)\right] \omega_{n i}=I_{n k}^{\prime}+I_{2} .
\end{gathered}
$$

Покажем, что

$$
P\left\{\sup _{k}\left|I_{2}\right|>\varepsilon\right\} \rightarrow 0, \quad n \rightarrow \infty .
$$

Здесь имеет место следующая оценка:

$$
P\left\{\sup _{k}\left|I_{2}\right|>\varepsilon\right\} \leqslant P\left\{\sup _{k}\left|\zeta_{n k}\right|>N\right\}+\frac{4}{\varepsilon^{2}} M\left(\sum_{i=0}^{k^{n-1}} \chi_{\left|\zeta_{n i}\right| \leqslant N} \times\right.
$$




$$
\begin{gathered}
\left.X\left|f^{\prime}{ }_{n}\left(\xi_{n i}\right)\left[\beta_{n i}-\sigma_{n}\left(\xi_{n i}\right)\right] \omega_{n i}\right|\right)^{2} \leqslant P\left\{\sup _{k}\left|\zeta_{n k}\right|>N\right\}+ \\
+\frac{4}{\varepsilon^{2}} K_{N_{0 \leqslant i \leqslant k_{n}-1}^{2}}\left(M\left[\frac{\beta_{n i}-\sigma_{n}\left(\xi_{n i}\right)}{\sigma_{n}\left(\xi_{n i}\right)}\right]^{4} \chi_{\left|\xi_{n i}\right| \leqslant N}\right)^{1 / 2} \times \\
\times\left(\sum_{i=0}^{k_{n}-1} \Delta t_{n i}\right)^{1 / 2} \rightarrow 0, \quad n \rightarrow \infty, \quad t \in[0,1],
\end{gathered}
$$

в силу условий теоремы 1 .

Далее, оценим

$$
I_{3}=\sum_{i=0}^{k} f_{n}^{\prime \prime}\left(\xi_{n i}\right)\left(\alpha_{n i} \Delta t_{n i}\right)^{2} .
$$

Так как

$$
\left(\alpha_{n i}\right)^{2} \leqslant 2\left(\alpha_{n i}-a_{n}\left(\xi_{n i}\right)\right)^{2}+2 a_{n}^{2}\left(\xi_{n i}\right),
$$

то

$$
\begin{gathered}
\left|I_{3}\right| \leqslant \sum_{i=0}^{k}\left|f_{n}^{\prime \prime}\left(\xi_{n i}\right)\right| a_{n}^{2}\left(\xi_{n i}\right) \Delta t_{n i}^{2}+ \\
+2 \sum_{i=0}^{k}\left|f_{n}^{\prime \prime}\left(\xi_{n i}\right)\right|\left[\alpha_{n i}-a_{n}\left(\xi_{n i}\right)\right]^{2} \Delta t_{n i}^{2}=I_{3}^{\prime}+I_{3}^{\prime \prime},
\end{gathered}
$$

$\sup _{k} \mid I^{\prime}{ }_{3} \rightarrow 0$ по вероятности, $n \rightarrow \infty \quad$ (см. доказательство теоремы 1 $\left.\left[{ }^{2}\right]\right)$. Поскольку

$$
\left|f_{n}^{\prime \prime}(x)\right|=\left|f_{n}^{\prime}(x) \sigma_{n}(x)\right|\left|\frac{a_{n}(x)}{\sigma_{n}(x)}\right| \frac{2}{\sigma_{n}^{2}(x)},
$$

то в силу условия 1) теоремы 1

$$
\left|f_{n}^{\prime \prime}(x)\right| \leqslant K_{N}\left|\frac{a_{n}(x)}{\sigma_{n}(x)}\right| \frac{1}{\sigma_{n}^{2}(x)} .
$$

Тогда

$$
\begin{aligned}
& P\left\{\sup _{k}\left|I_{3}^{\prime \prime}\right|>\varepsilon\right\} \leqslant P\left\{\sup _{k}\left|\xi_{n k}\right|>N\right\}+\frac{2}{\varepsilon} M\left(\sum_{i=0}^{k_{n}-1} \chi_{\left|\zeta_{n i}\right| \leqslant N} K_{N} \times\right. \\
\times & \left.\left|\frac{a_{n}\left(\xi_{n i}\right)}{\sigma_{n}\left(\xi_{n i}\right)}\right|\left[\frac{a_{n i}-a_{n}\left(\xi_{n i}\right)}{\sigma_{n}\left(\xi_{n i}\right)}\right]^{2}\left(\Delta t_{n i}\right)^{2}\right) \leqslant P\left\{\sup _{k}\left|\zeta_{n k}\right|>N\right\}+ \\
+ & \frac{2}{\varepsilon} K_{N} \lambda_{n}^{2} \sup _{\left|f_{n}(x)\right| \leqslant N}\left|\frac{a_{n}(x)}{\sigma_{n}(x)}\right| \sup _{i} M\left[\frac{\alpha_{n i}-a_{n}\left(\xi_{n i}\right)}{\sigma_{n}\left(\xi_{n i}\right)}\right]^{2} \chi_{\left|\zeta_{n i}\right| \leqslant N},
\end{aligned}
$$

а эта величина стремится к $0, n \rightarrow \infty$, в силу условий теоремы 1 . Следовательно,

$$
\sup _{k}\left|I_{3}\right| \rightarrow 0 \text { по вероятности, } n \rightarrow \infty .
$$

Рассмотрим теперь 


$$
\begin{aligned}
& I_{4}=\sum_{i=0}^{k} f_{n}^{\prime \prime}\left(\xi_{n i}\right) \alpha_{n i} \beta_{n i} \omega_{n i} \Delta t_{n i} \\
& I_{4}=\sum_{i=0}^{k} f_{n}^{\prime \prime}\left(\xi_{n i}\right)\left[\alpha_{n i}-a_{n}\left(\xi_{n i}\right)\right] \beta_{n i} \omega_{n i} \Delta t_{n i}+\sum_{i=0}^{k} f_{n}^{\prime \prime}\left(\xi_{n i}\right) a_{n}\left(\xi_{n i}\right) X \\
& \times \beta_{n i} \omega_{n i} \Delta t_{n i}=I_{4}^{\prime}+I_{4}^{\prime \prime} \\
& P\left\{\sup _{k}\left|I_{4}^{\prime}\right|>\varepsilon\right\} \leqslant P\left\{\sup _{k}\left|\xi_{n k}\right|>N\right\}+\frac{4}{\varepsilon^{2}} K_{N}^{2} \sum_{i=0}^{k_{n}-1} M\left|\frac{a_{n}\left(\xi_{n i}\right)}{\sigma_{n}\left(\xi_{n i}\right)}\right|^{2} \times \\
& \times\left|\frac{\alpha_{n i}-a_{n}\left(\xi_{n i}\right)}{\sigma_{n}\left(\xi_{n i}\right)}\right|^{2} \chi_{\left|\xi_{n i}\right| \leqslant N}\left[\frac{\beta_{n i}}{\sigma_{n}\left(\xi_{n i}\right)}\right]^{2}\left(\Delta t_{n i}\right)^{3} \leqslant P\left\{\sup _{k}\left|\zeta_{n k}\right|>N\right\}+ \\
& +2 K_{N}^{2} \sum_{i=0}^{k_{n}-1} M\left|\frac{a_{n}\left(\xi_{n i}\right)}{\sigma_{n}\left(\xi_{n i}\right)}\right|^{2}\left|\frac{\alpha_{n i}-a_{n}\left(\xi_{n i}\right)}{\sigma_{n}\left(\xi_{n i}\right)}\right|^{2}\left|\frac{\beta_{n i}-\sigma_{n}\left(\xi_{n i}\right)}{\sigma_{n}\left(\xi_{n i}\right)}\right|^{2} \times \\
& \chi_{\left|\xi_{n i}\right| \leqslant N}\left(\Delta t_{n i}\right)^{3}+2 K_{N}^{2} \sum_{i=0}^{k_{n}-1} M\left|\frac{a_{n}\left(\xi_{n i}\right)}{\sigma_{n}\left(\xi_{n i}\right)}\right|^{2}\left|\frac{\alpha_{n i}-a_{n}\left(\xi_{n i}\right)}{\sigma_{n}\left(\xi_{n i}\right)}\right|^{2} \times \\
& X\left(\Delta t_{n i}\right)^{3} \chi_{\left|\zeta_{n i}\right| \leqslant N} \leqslant P\left\{\sup _{k}\left|\zeta_{n k}\right|>N\right\}+2 K_{N}^{2} \lambda_{n}^{2} \times \\
& \times \sup _{\left|f_{n}(x)\right| \leqslant N}\left|\frac{a_{n}(x)}{\sigma_{n}(x)}\right|^{2} \sup _{i} \sqrt{M\left|\frac{\alpha_{n i}-a_{n}\left(\xi_{n i}\right)}{\sigma_{n}\left(\xi_{n i}\right)}\right|^{4}} \sqrt{M\left|\frac{\beta_{n i}-\sigma_{n}\left(\xi_{n i}\right)}{\sigma_{n}\left(\xi_{n i}\right)}\right|^{4}} \times \\
& \times \sum_{i=0}^{k_{n}-1} \Delta t_{n i}+2 K_{N}^{2} \lambda_{n}^{2} \sup _{\left|f_{n}(x)\right| \leqslant N}\left|\frac{a_{n}(x)}{\sigma_{n}(x)}\right|^{2} \sup _{i} \sqrt{M\left|\frac{\alpha_{n i}-a_{n}\left(\xi_{n i}\right)}{\sigma_{n}\left(\xi_{n i}\right)}\right|^{4}},
\end{aligned}
$$

а эта величина стремится к $0, n \rightarrow \infty$, что следует из условий теоремы 1 . Значит,

$$
\sup _{k}\left|I_{4}^{\prime}\right| \rightarrow 0 \text { по вероятности, } n \rightarrow \infty \text {. }
$$

Далее,

$$
\begin{aligned}
& P\left\{\sup _{k}\left|I_{4}^{\prime \prime}\right|>\varepsilon\right\} \leqslant P\left\{\sup _{k}\left|\zeta_{n k}\right|>N\right\}+P\left\{\sup _{k} \mid \sum_{i=0}^{k} f_{n}^{\prime \prime}\left(\xi_{n i}\right) X\right. \\
& \left.X a_{n}\left(\xi_{n i}\right) \beta_{n i} \omega_{n i} \Delta t_{n i} \chi_{\left|\xi_{n i}\right| \leqslant N} \mid>\frac{\varepsilon}{2}\right\} \leqslant P\left\{\sup _{k}\left|\xi_{n k}\right|>N\right\}+ \\
& +\frac{4}{\varepsilon^{2}} K_{N}^{2} \lambda_{n}^{2} \sup _{\mid f_{n}(x) i \leqslant N}\left|\frac{a_{n}(x)}{\sigma_{n}(x)}\right|^{4} \sum_{i=0}^{k_{n}-1} M\left[\frac{\beta_{n i}}{\sigma_{n}\left(\xi_{n i}\right)}\right]^{2} \Delta t_{n i} \leqslant \\
& \leqslant P\left\{\sup _{k}\left|\zeta_{n k}\right|>N\right\}+\frac{8}{\varepsilon^{2}} K_{N}^{2} \lambda_{n}^{2} \sup _{\left|f_{n}(x)\right| \leqslant N}\left|\frac{a_{n}(x)}{\sigma_{n}(x)}\right|^{4} X
\end{aligned}
$$




$$
\times \sum_{i=0}^{k_{n}-1} M\left[\frac{\beta_{n i}-\sigma_{n}\left(\xi_{n i}\right)}{\sigma_{n}\left(\xi_{n i}\right)}\right]^{2} \Delta t_{n i}+\lambda_{n}^{2} \sup _{\left|f_{n}(x)\right| \leqslant N}\left|\frac{a_{n}(x)}{\sigma_{n}(x)}\right|^{4} \sum_{i=0}^{k_{n}-1} \Delta t_{n i},
$$

а последняя сумма стремится к $0, n \rightarrow \infty$. Следовательно,

$$
\sup _{k}\left|I_{4}\right| \rightarrow 0 \quad \text { по вероятности, } n \rightarrow \infty .
$$

Оценим теперь

$$
I_{5}=\sum_{i=0}^{k} f_{n}^{\prime \prime}\left(\xi_{n i}\right)\left[\beta_{n i}^{2}-\sigma_{n}^{2}\left(\xi_{n i}\right)\right] \Delta t_{n i} .
$$

Поскольку

$$
\begin{aligned}
& \left|\sum_{i=0}^{k} f_{n}^{\prime \prime}\left(\xi_{n i}\right)\left[\beta_{n i}^{2}-\sigma_{n}^{2}\left(\xi_{n i}\right)\right] \Delta t_{n i}\right| \leqslant 2 K_{N} \sup _{\left|f_{n}(x)\right| \leqslant N}\left|\frac{a_{n}(x)}{\sigma_{n}(x)}\right| X \\
& X \sup _{k}\left|\frac{\beta_{n k}-\sigma_{n}\left(\xi_{n k}\right)}{\sigma_{n}\left(\xi_{n k}\right)}\right|^{2} \sum_{i=0}^{k} \Delta t_{n i}+4 K_{N} \sup _{k}\left[\frac{\beta_{n k}-\sigma_{n}\left(\xi_{n k}\right)}{\sigma_{n}\left(\xi_{n k}\right)}\right],
\end{aligned}
$$

To

$$
\begin{gathered}
P\left\{\sup _{k}\left|I_{5}\right|>\varepsilon\right\} \leqslant P\left\{\sup _{k}\left|\xi_{n k}\right|>N\right\}+\frac{8}{\varepsilon} K_{N}[1+ \\
\left.+\sup _{\left|f_{n}(x)\right| \leqslant N} \frac{a_{n}(x)}{\sigma_{n}(x)}\right] \sup _{k} M\left[\frac{\beta_{n k}-\sigma_{n}\left(\xi_{n k}\right)}{\sigma_{n}\left(\xi_{n k}\right)}\right] \chi_{\left|\xi_{n k}\right| \leqslant N} \rightarrow 0, \quad n \rightarrow \infty .
\end{gathered}
$$

Следовательно,

$$
\sup _{k}\left|I_{5}\right| \rightarrow 0 \quad \text { по вероятности, } n \rightarrow \infty .
$$

И, наконец, осталось оценить

$$
\begin{gathered}
I_{6}=\sum_{i=0}^{k} f_{n}^{\prime \prime}\left(\xi_{n i}\right) \beta_{n i}^{2}\left(\omega_{n i}^{2}-\Delta t_{n i}\right) . \\
P\left\{\sup _{k}\left|I_{6}\right|>\varepsilon\right\} \leqslant P\left\{\sup _{k}\left|\xi_{n k}\right|>N\right\}+\frac{4}{\varepsilon^{2}} K_{N}^{2} \times \\
\times \sup _{\left|f_{n}(x)\right| \leqslant N}\left|\frac{a_{n}(x)}{\sigma_{n}(x)}\right|^{2}\left\{2 \sum_{i=0}^{k_{n}-1}\left[\frac{\Delta \xi_{n i}}{\sigma_{n}\left(\xi_{n i}\right)}\right]^{4}+2 \sum_{i=0}^{k_{n}-1}\left[\frac{a_{n i}-a_{n}\left(\xi_{n i}\right)}{\sigma_{n}\left(\xi_{n i}\right)}\right]^{4} \times\right. \\
\times\left(\Delta t_{n i}\right)^{4}+2 \sum_{i=0}^{k_{n}-1}\left[\frac{a_{n}\left(\xi_{n i}\right)}{\sigma_{n}\left(\xi_{n i}\right)}\right]^{4}\left(\Delta t_{n i}\right)^{4}+2 \sum_{i=0}^{k_{n}-1}\left[\frac{\beta_{n i}-\sigma_{n i}\left(\xi_{n i}\right)}{\sigma_{n}\left(\xi_{n i}\right)}\right]^{4} \times \\
\left.\times\left(\Delta t_{n i}\right)^{2}+2 \lambda_{n} \sum_{i=0}^{k} \Delta t_{n i}\right\} \rightarrow 0, \quad n \rightarrow \infty .
\end{gathered}
$$

При оценке использовались соотношения (18) и

$$
\left(\omega_{n i}\right)^{4} \leqslant \frac{\left(\Delta \xi_{n i}\right)^{4}}{\beta_{n i}^{4}}+\frac{\left(\alpha_{n i}\right)^{4}}{\beta_{n i}^{4}}\left(\Delta t_{n i}\right)^{4}
$$


Из этого следует:

$$
\sup _{k}\left|I_{6}\right| \rightarrow 0 \quad \text { по вероятности, } n \rightarrow \infty .
$$

Покажем теперь, что

$$
I^{\prime}{ }_{n k}=\sum_{i=0}^{k} f_{n}^{\prime}\left(\xi_{n i}\right) \sigma_{n}\left(\xi_{n i}\right) \omega_{n i} \stackrel{\text { слабо }}{\longrightarrow} \int_{0}^{t} \sigma_{0}(\xi(s)) d w(s), \quad n \rightarrow \infty .
$$

Для доказательства этого факта придется воспользоваться некоторыми леммами, доказательство которых, аналогичное доказательству соответствующих лемм в $\left[{ }^{2}\right]$, труда не представляет.

Л ем а 1. Процесс $w_{n}(t)$ при $n \rightarrow \infty$ слабо сходится к винеровскому процессу.

Здесь имеется в виду, что $w_{n}(t)=\sum_{t_{n k}<t} \omega_{n k}$.

Л емм а 2. Для любого конечного числа точек $x_{j}, j=\overline{1, m}$,

$$
\lim _{\varepsilon \rightarrow 0} \overline{\lim }_{n \rightarrow \infty}^{k_{n}{ }_{n}-1} P\left\{\left.\right|_{k=0}{ }_{\llcorner n k}-x_{j} \mid \leqslant \varepsilon, j=\overline{1, m}\right\} \Delta t_{n k}=0 .
$$

Л ем м а 3. Пусть $|g(x)| \leqslant C$. Тогда для любых $t^{\prime}<t^{\prime \prime}$

$$
\varlimsup_{n \rightarrow \infty} M\left|\sum_{t^{\prime}<t_{n k}<t^{\prime \prime}} g\left(\xi_{n k}\right) \omega_{n k}\right|^{4} \leqslant 6 C^{4}\left[t^{\prime \prime}-t^{\prime}\right]^{2} \text {. }
$$

Вернемся к доказательству теоремы 1. Поскольку

$$
\zeta_{n}(t)=\zeta_{n k}+\frac{t-t_{n k}}{t_{n k+1}-t_{n k}}\left(\zeta_{n k+1}-\zeta_{n k}\right), \quad t \in\left[t_{n k}, t_{n k+1}\right],
$$

a

$$
\begin{aligned}
& \zeta_{n k+1}=\zeta_{n 0}+I_{1}+I_{n k}^{\prime}+I_{2}+I_{3}+I_{4}+I_{5}+I_{6}+R_{n}, \\
& \sup _{k}\left|I_{i}\right| \stackrel{P}{\rightarrow} 0, n \rightarrow \infty, i=\overline{1,6},\left|R_{n}\right| \stackrel{P}{\rightarrow} 0, n \rightarrow \infty,
\end{aligned}
$$

по условиям теоремы 1 , то легко показать, что

$$
\begin{gathered}
\lim _{c \rightarrow \infty} \varlimsup_{n \rightarrow \infty} P\left\{\left|\zeta_{n}(t)\right|>C\right\}=0, \\
\lim _{h \rightarrow 0} \varlimsup_{n \rightarrow \infty} \sup _{\left|t^{\prime}-t^{\prime \prime}\right| \leqslant h} P\left\{\left|\zeta_{n}\left(t^{\prime}\right)-\zeta_{n}\left(t^{\prime \prime}\right)\right|>\varepsilon\right\}=0,
\end{gathered}
$$

для любого $\varepsilon>0$. Аналогичные соотношения выполняются и для процесса $w_{n}(t)$. Поэтому процесс $\left(\zeta_{n}(t), w_{n}(t)\right)$ обладает свойством компактности $\left.{ }^{1}{ }^{1}\right]$ и будем считать, что для любой подпоследовательности $n_{k} \rightarrow \infty$ существует подпоследовательность $n_{k}^{\prime} \rightarrow \infty$ такая, что $\zeta_{n^{\prime}}(t) \stackrel{P}{\rightarrow} \zeta(t), w_{n^{\prime}}(t) \stackrel{P}{\rightarrow} w(t)$, где $\zeta(t)$ и $w(t)$ - некоторые случайные процессы. На основании леммы 1 w(t) является винеровским процессом. Из соотношений (24):

$$
\sup _{0 \leqslant t \leqslant 1}\left|\zeta_{n^{\prime}}(t)-\zeta_{n^{\prime}}(0)-\int_{0}^{t} \hat{f}_{n}^{\prime}\left(\zeta_{n^{\prime}}(s)\right) d w_{n^{\prime}}(s)\right| \stackrel{P}{\rightarrow} 0, n^{\prime} \rightarrow \infty,
$$


$\hat{f}_{n}^{\prime}(x)=f_{n}^{\prime}\left(\varphi_{n}(x)\right) \sigma_{n}\left(\varphi_{n}(x)\right), \quad \varphi_{n}(x)-$ функция, обратная к функции $f_{n}(x)$. Так как

$$
\begin{gathered}
P\left\{\sup _{0 \leqslant t \leqslant 1}\left|\int_{0}^{t} \hat{f}_{n}^{\prime}\left(\zeta_{n}(s)\right) d w_{n}(s)-\int_{0}^{t} \sigma_{0}\left(\zeta_{n}(s)\right) d w_{n}(s)\right|>\varepsilon\right\} \leqslant \\
\leqslant \frac{4}{\varepsilon^{2}} \sum_{k=0}^{k} M\left|\hat{f}_{n}^{\prime}{ }_{n}\left(\zeta_{n k}\right)-\sigma_{0}\left(\zeta_{n k}\right)\right|^{2} \chi_{N}\left(\zeta_{n k}\right) \Delta t_{n k}+P\left\{\sup _{0 \leqslant t \leqslant 1}\left|\zeta_{n}(t)\right|>N\right\}
\end{gathered}
$$

для любых $\varepsilon>0$ и $N>0$, то

$$
\sup _{0 \leq t \leq 1}\left|\int_{0}^{t} \hat{f}^{\prime}{ }_{n}\left(\zeta_{n}(s)\right) d w_{n}(s)-\int_{0}^{t} \sigma_{0}\left(\zeta_{n}(s)\right) d w_{n}(s)\right| \stackrel{P}{\rightarrow} 0, n \rightarrow \infty,
$$

и далее доказательство проходит аналогично доказательству теоремы 1 в $\left[{ }^{2}\right]$.

Итак, получаем:

$$
\zeta(t)=\zeta_{0}+\int_{0}^{t} \sigma_{0}(\zeta(s)) d w(s)
$$

и $F\left(\zeta_{n^{\prime}{ }_{k}}(t)\right) \rightarrow F(\zeta(t))$ с вероятностью $1, n^{\prime}{ }_{k} \rightarrow \infty$, для любого непрерывного на $C_{[0,1]}$ функционала $F$. Из единственности $(26)$ решения уравнения (27) и произвольности подпоследовательности $n_{k}$ получаем доказательство теоремы 1.

Т е орем а 2. Пусть выполняются условия теоремы 1 , а последовательность функций $g_{n}(x)$ такая, что для любой постоянной $N>0$

$$
\begin{gathered}
\lim _{n \rightarrow \infty}\left\{\lambda_{n}\left[\sup _{\left|f_{n}(x)\right| \leqslant N} g_{n}^{2}(x)+\sup _{\left|f_{n}(x)\right| \leqslant N}\left|g_{n}(x)\right|\left(\frac{a_{n}(x)}{\sigma_{n}(x)}\right)^{2}\right]+\right. \\
\left.+\sup _{\left|f_{n}(x)\right| \leqslant N} g_{n}^{2}(x) \sum_{k=0}^{k_{n}-1} M\left[\frac{\Delta \xi_{n k}}{\sigma_{n}\left(\xi_{n k}\right)}\right]^{4} \chi_{N}\left(\xi_{n k}\right)\right\}=0, \\
\sup _{\left|f_{n}(x)\right| \leqslant N}\left|\int_{0}^{x} \frac{g_{n}(u)}{f_{n}^{\prime}(u) \sigma_{n}^{2}(u)} d u\right| \leqslant \widetilde{K}_{N}, \\
\lim _{n \rightarrow \infty} \sum_{k=0}^{k_{n}-1} M\left|\int_{\xi_{n k}}^{\xi_{n h+1} x} \int_{n k}\left[G_{n}^{\prime \prime}(u)-G_{n}^{\prime \prime}\left(\xi_{n k}\right)\right] d u d x\right| \chi_{N}\left(\zeta_{n k}\right)=0,
\end{gathered}
$$

$\varepsilon \partial е$

$$
G_{n}(x)=2 \int_{0}^{x} f_{n}^{\prime}(u) \int_{0}^{u} \frac{g_{n}(u)}{f_{n}^{\prime}(v) \sigma_{n}^{2}(v)} d v d u,
$$

кроме того, существует функция $g(x)$, для которой

$$
\int_{0}^{x} \frac{g_{n}\left(\varphi_{n}(u)\right)}{\left[f_{n}^{\prime}\left(\varphi_{n}(u)\right) \sigma_{n}\left(\varphi_{n}(u)\right)\right]^{2}} d u \rightarrow g(x)
$$

$\left(\varphi_{n}(x)-\right.$ функция, обратная $\kappa$ функции $\left.f_{n}(x)\right)$ при $n \rightarrow \infty$ равномерно в каждом компакте, не содержащем определенного (без точек сәуще- 
ния) множества точек. Тогда случайная ломаная $\eta_{n}(t)$ с вериинами в точках $\left(t_{n k}, \sum_{i=0}^{k} g_{n}\left(\xi_{n i}\right) \Delta t_{n i}\right)$ при $n \rightarrow \infty$ слабо сходится $\kappa$ процессу

$$
\eta(t)=2\left[\int_{\zeta_{0}}^{\zeta(t)} g(u) d u-\int_{0}^{t} g(\zeta(s)) d \zeta(s)\right],
$$

где $\zeta(t)$ - решение уравнения

$$
\zeta(t)=\zeta_{0}+\int_{0}^{t} \sigma_{0}(\zeta(s)) d w(s),
$$

и для всякого непрерывного на $C_{[0,1]}$ функционала $F$ распределение $F\left(\eta_{n}(t)\right)$ сходится $\kappa$ распределению $F(\eta(t))$.

Д ок а з а тел в с т в о. $\Delta \xi_{n k}=\alpha_{n k} \Delta t_{n k}+\beta_{n k} \omega_{n k}$ (см. доказательство теоремы 1). В силу вида $G_{n}(x)$ имеем $G_{n}^{\prime}(x) a_{n}(x)+1 / 2 G_{n}^{\prime \prime}(x) \sigma_{n}^{2}(x)=$ $=g(x)$.

Поэтому

$$
\begin{aligned}
& \sum_{i=0}^{k} g_{n}\left(\xi_{n i}\right) \Delta t_{n i}=G_{n}\left(\xi_{n k+1}\right)-G_{n}\left(\xi_{n 0}\right)-\sum_{i=0}^{k} G_{n}^{\prime}\left(\xi_{n i}\right) \times \\
& X\left[\alpha_{n i}-a_{n}\left(\xi_{n i}\right)\right] \Delta t_{n i}-\sum_{i=0}^{i} G_{n}^{\prime}\left(\xi_{n i}\right) \beta_{n i\left(\omega_{n i}\right.}-\frac{1}{2} \sum_{i=0}^{h} G_{n}^{\prime \prime}\left(\xi_{n i}\right) X \\
& \left.\times\left(\alpha_{n i} \Delta t_{n i}\right)^{2}-\sum_{i=0}^{h} G_{n}^{\prime \prime}\left(\xi_{n i}\right) \alpha_{n i} \beta_{n i(}\right)_{n i} \Delta t_{n i}-\frac{1}{2} \sum_{i=0}^{h} G_{n}^{\prime \prime}\left(\xi_{n i}\right) X \\
& \times\left[\beta_{n i}^{2}-\sigma_{n}^{2}\left(\xi_{n i}\right)\right] \Delta t_{n i}-\frac{1}{2} \sum_{i=0}^{k} G_{n}^{\prime \prime}\left(\xi_{n i}\right) \beta_{n i}^{2}\left(\omega_{n i}^{2}-\Delta t_{n i}\right)- \\
& -\sum_{i=0}^{k} \int_{\xi_{n i}}^{\xi_{n+1}} \int_{\xi_{n i}}^{x}\left[G_{n}^{\prime \prime}(u)-G_{n}^{\prime \prime}\left(\xi_{n i}\right)\right] d u d x=G_{n}\left(\xi_{n k+1}\right)- \\
& -G_{n}\left(\xi_{n 0}\right)-J_{1}-J_{n k}-J_{2}-J_{3}-J_{4}-J_{5}-R_{n} \text {. } \\
& \left|R_{n}\right| \stackrel{P}{\rightarrow} 0, \quad n \rightarrow \infty, \\
& \sup _{k}\left|J_{i}\right| \stackrel{P}{\rightarrow} 0, \quad n \rightarrow \infty, \quad i=\overline{1,5},
\end{aligned}
$$

в силу условий теоремы 2. Показано это может быть точно таким же методом, что и при доказательстве теоремы 1. Например,

$$
\begin{gathered}
\sup _{k}\left|J_{1}\right|=\sup _{k}\left|2 \widetilde{K}_{N} \sum_{i=0}^{k} f_{n}^{\prime}\left(\xi_{n i}\right)\left[\alpha_{n i}-a_{n}\left(\xi_{n i}\right)\right] \Delta t_{n i}\right| \leqslant \\
\leqslant \sup _{k}\left|I_{1}\right| \stackrel{P}{\rightarrow} 0, \quad n \rightarrow \infty .
\end{gathered}
$$

Поэтому

$$
\sum_{i=0}^{k} g_{n}\left(\xi_{n i}\right) \Delta t_{n i}=G_{n}\left(\xi_{n k+1}\right)-G_{n}\left(\xi_{n 0}\right)-\sum_{i=0}^{k} G_{n}^{\prime}\left(\xi_{n i}\right) \beta_{n i()_{n i}}-o(1),
$$




$$
o(1) \rightarrow 0, \quad n \rightarrow \infty
$$

Следовательно,

$$
\sum_{i=0}^{k} g_{n}\left(\xi_{n i}\right) \Delta t_{n i}=G_{n}\left(\xi_{n k+1}\right)-G_{n}\left(\xi_{n 0}\right)-\sum_{i=0}^{k} G_{n}^{\prime}\left(\xi_{n i}\right) \sigma_{n}\left(\xi_{n i}\right) \omega_{n i}-o(1),
$$

поскольку

$$
\sup _{k}\left|\sum_{i=0}^{k} G_{n}^{\prime}\left(\xi_{n i}\right)\left[\beta_{n i}-\sigma_{n}\left(\xi_{n i}\right)\right] \omega_{n i}\right| \stackrel{P}{\rightarrow} 0, \quad n \rightarrow \infty,
$$

в силу тех же соображений, что и при доказательстве теоремы 1. Далее доказательство совпадает с доказательством теоремы $2\left[{ }^{2}\right]$. В самом деле, случайная ломаная $\eta_{n}(t)$ представима в виде

$$
\begin{aligned}
& \eta_{n}(t)=\sum_{i=0}^{h} g_{n}\left(\xi_{n i}\right) \Delta t_{n i}+\left[t-t_{n k}\right] g_{n}\left(\xi_{n k}\right)=G_{n}\left(\hat{\zeta}_{n}(t)\right)- \\
& -G_{n}\left(\hat{\zeta}_{n}(0)\right)+G_{n}\left(\hat{\zeta}_{n}\left(t_{n k+1}\right)\right)-G_{n}\left(\hat{\zeta}_{n}(t)\right)-\int_{0}^{t} G_{n}^{\prime}\left(\hat{\zeta}_{n}(s)\right) \times \\
& \times \sigma_{n}(\hat{\zeta}(s)) d w_{n}(s)-\left(J_{1}+J_{2}+J_{3}+J_{4}+J_{5}+\sum_{i=0}^{k} G_{n}^{\prime}\left(\xi_{n i}\right) \times\right. \\
& \times\left[\beta_{n i}-\sigma_{n}\left(\xi_{n i}\right)\right]\left(\omega_{n i}\right)-\left[t-t_{n k}\right] g\left(\xi_{n k}\right), \quad t \in\left[t_{n k}, t_{n k+1}\right] .
\end{aligned}
$$

Учитывая (36) и (37), получаем

$$
\begin{gathered}
\sup _{0 \leqslant t \leqslant 1} \mid \eta_{n}(t)-G_{n}\left(\hat{\zeta}_{n}(t)\right)-G_{n}\left(\hat{\zeta}_{n}(0)\right)-\int_{0}^{t} G_{n}^{\prime}\left(\hat{\zeta}_{n}(s)\right) \times \\
\times \sigma_{n}\left(\hat{\zeta}_{n}(s)\right) d w_{n}(s) \mid \stackrel{P}{\rightarrow} 0, \quad n \rightarrow \infty,
\end{gathered}
$$

причем $\quad \hat{\zeta}_{n}(t)=\varphi_{n}\left(\zeta_{n}(t)\right)$.

Поэтому (см. $\left[{ }^{2}\right]$ доказательство теоремы 2):

$$
\sup _{0 \leqslant t \leqslant 1}\left|\eta_{n^{\prime}}(t)-2 \int_{\zeta(0)}^{\zeta(t)} g(u) d u-2 \int_{0}^{t} g(\zeta(s)) d \zeta(s)\right| \stackrel{P}{\rightarrow} 0, \quad n^{\prime} \rightarrow \infty .
$$

Из произвольности последовательности $n^{\prime}$ следует доказательство теоремы.

В заключение автор приносит благодарность Г. Л. Кулиничу за помощь при работе над настоящей статьей.

\section{ЛИТЕРАТУ РА}

1. С короход А. В., Исследования по теории случайных процессов, Киев, 1961.

2. К улинич Г. Л., В сб.: Теория вероятностей и математическая статистика, вып. 12, Киев, 1975.

Таллинский политехнический институт
Поступила в редакщию

15/I 1976 
Jelena ROOS

\section{SUVALISELT MUUTUVATE OHEMÕTTMELISTE JUHUSLIKE SUURUSTE SEERIATE JADA PIIRTEOREEMID}

Esitatakse juhuslike suuruste seeria koondumise piisavad tingimused, mis on rakendatavad katkevate kordajate difusiooniprotsessis, ning tingimused, mille alusel seeriate jada teatud klassi funktsionaalid koonduvad piirprotsessist teatud funktsionaaliks.

Yelena ROOS

\section{LIMIT THEOREMS FOR SEQUENCE OF SERIES OF ONE-DIMENSIONAL RANDOM VARIABLES WITH ARBITRARY DEPENDENCE CHARACTER}

In the paper the convergence of sequences of one-dimensional random variables with arbitrary dependence character to diffusion process with discontinuous coefficients is investigated. The convergence of some class of functionals dependent on sequence of series to some functionals of the limit process is studied. 